\title{
EL ESPACIO COMO EJE VERTEBRADOR EN LA CREACIÓN DEL UNIVERSO FICTICIO GALDOSIANO: UN ESTUDIO DE CORPUS
}

\author{
SPATIAL REFERENCES AS A STRUCTURING DEVICE IN THE \\ CREATION OF FICTIONAL UNIVERSES \\ IN PÉREZ GALDÓS'S NOVELS: \\ A STUDY OF THE COURPUS
}

\author{
Guadalupe NIETO CABALLERO \\ Universidad de Extremadura \\ gnieto@unex.es
}

\begin{abstract}
Resumen: En este artículo se analiza el valor del espacio como eje vertebrador en la creación de los universos ficticios en las novelas de Benito Pérez Galdós. Para ello hemos utilizado un enfoque de estilística corpus. El análisis se articula en torno a clusters identificados gracias al procesamiento de los textos con un software de concordancias, que ha revelado patrones tanto formales como funcionales que tradicionalmente han pasado desapercibidos para la crítica especializada. El estudio pretende arrojar luz sobre los hábitos estilísticos de Galdós en su tratamiento del espacio, uno de los aspectos por los que goza de mayor reconocimiento.
\end{abstract}

Palabras clave: Galdós. Referencias espaciales. Universo ficticio. Estilística de corpus. 


\begin{abstract}
In this article, we analyze the role of space as one the aspects on which the creation of fictional universes in Benito Pérez Galdós's novels are built. To do so, we have used a corpus-stylistic approach. The analysis focuses on clusters retrieved with a software tool, which have revealed both formal and functional patterns that have traditionally gone unnoticed by literary critics. The study aims to shed some light on Galdós's stylistic habits in his treatment of space, one of the aspects for which he is best known.
\end{abstract}

Key Words: Galdós. Spatial references. Fictional universe. Corpus stylistics.

\title{
1. INTRODUCCIÓN
}

En este artículo se analizan patrones formales y funcionales relacionados con la configuración del espacio en los universos ficticios de la producción narrativa de Benito Pérez Galdós. El estudio del espacio en la obra de Galdós resulta importante, además de por su interés como documento de una época, por ser un elemento fundamental de las novelas en el plano simbólico, pues se encuentra cargado de significado (véase apartado 2). Desde un punto de vista estilístico, por otro lado, observar los criterios de caracterización del espacio del referente y su función (Álvarez Méndez, 2002: 70) resulta igualmente importante, ya que en él se advierten mecanismos y hábitos que definen el estilo del autor. En este estudio nos fijamos en el uso repetido de construcciones que contienen referencias espaciales en la producción Galdós, construcciones que analizamos desde el punto de vista de las funciones que desempeñan en la construcción de los universos ficticios que el autor nos plantea. Para ello, hemos utilizado un enfoque de corpus (Mahlberg, 2016).

Mediante una lectura atenta, como tradicionalmente ha venido haciendo la crítica especializada, se pueden apreciar aspectos relevantes 
estilísticamente relacionados con el espacio. Sin embargo, en el caso de un autor cuya producción está formada por más de setenta novelas y más de seis millones de palabras, identificar patrones formales y funcionales resulta una tarea prácticamente imposible. Esta es precisamente la vía que abren los enfoques de corpus, cuyo potencial analítico nos permite descubrir "patterns that we as readers may not be aware of, although such patterns might still contribute to the effects we perceive" (Mahlberg, 2013: 27). En el caso de este artículo, veremos cómo el dominio del espacio visual y del movimiento con el que "se prefigura el ritmo cinematográfico" de muchas de sus obras (Arroyo Díez, 2011: 366) se cimienta sobre el uso repetido de construcciones con referencias espaciales que no han sido analizadas de forma sistemática por la crítica especializada. En este sentido, podría decirse que este artículo pretende también contribuir a demostrar el potencial de este tipo de enfoques en los estudios literarios de obras en lengua española, y abrir así nuevas vías de análisis que hasta ahora no han sido exploradas en la exégesis de autores en este idioma.

\section{MARCO DE ESTUDIO: EL ESPACIO EN EL GÉNERO NARRATIVO Y EN LA OBRA DE GALDÓS}

El espacio, entendido como escenario en el que tiene lugar la acción, es una parte fundamental del texto novelesco (Álvarez Méndez, 2002) y un elemento que se encuentra en estrecha relación con el resto de componentes de la estructura formal. Junto con el tiempo, los personajes y los acontecimientos que tienen lugar en la historia, el espacio es uno de los pilares estructurales de la sintaxis narrativa: toda narración implica una serie de acontecimientos y personajes que se desarrollan en un tiempo y también en un espacio determinados (Zubiaurre, 2000: 20-21). La importancia del espacio en el género novelesco es tal que hay quienes llegan a distinguir entre tipologías de novelas como "novelas de situaciones" o "de espacio" (Valles Calatrava, 2008: 178). Si nos centramos en la novela realista española, el espacio se erige en un elemento de importancia cardinal en 
la ilusión de transparencia y el efecto de verosimilitud que domina este movimiento. Al ser la reproducción fiel del mundo exterior uno de los pilares del realismo (Martínez Carazo, 2006: 36-37), la actitud del novelista no puede ser sino objetiva e impersonal, tratando de describir los espacios de manera detallada y exacta. La descripción sistemática de los espacios que domina la novela realista otorga a las escenas, y sobre todo a los personajes que las protagonizan, un entorno físico corpóreo, que hace que la historia parezca real (Matzat, 2007). Además de este importante papel en la construcción del universo narrativo, la configuración espacial se revela como uno de los elementos con más posibilidades de interpretación (Zorán, 1984: 319). Así, las profundas y minuciosas descripciones de los espacios típicas de la novela realista dotan habitualmente a los escenarios de un importante contenido semántico que habla indirectamente de los personajes y contribuye a su definición. El espacio, por tanto, no solo sirve de telón de fondo a los personajes, sino que juega un papel estilístico que suele ir más allá de la realidad geográfica sobre la que se asientan los actantes y en la que llevan a cabo sus acciones.

Si nos centramos en la figura de Galdós, vemos que los distintos rasgos que combina su estilo se encuentran armonizados en un universo novelesco que resulta, ante todo, convincente. Este realismo es en gran medida el resultado de su tratamiento del espacio, descrito con minuciosidad en sus obras gracias a su conocida labor de documentación en la que anotaba datos de escenarios que trasladaba a sus novelas. Sin embargo, su magistral tratamiento del espacio no solo queda patente en precisas descripciones de los enclaves en los que se desarrollan las historias, sino que tiene implicaciones literarias que van más allá de la propia configuración de la escena. En muchas de sus novelas, el espacio adquiere un valor simbólico en relación con determinadas funciones que envuelven y llegan a definir la actuación de los personajes. En La de Bringas, por ejemplo, Galdós hace coincidir los sentimientos y las actitudes de los personajes con el espacio en el que se desarrolla la acción (López-Landy, 1979: 182). El espacio del teatro, por ejemplo, sirve para proyectar la vanidad y el afán por aparentar 
de Rosalía Bringas. En Fortunata y Jacinta, por su parte, el espacio interior del convento de las Micaelas representa una atmósfera opresiva marcada por el dominio de las religiosas. Lo mismo ocurre con Torquemada en la hoguera, donde el espacio interior representa la expresión de un ámbito opresor, pues es donde el usurero presencia cómo se consume la vida de su hijo. Finalmente, en El doctor Centeno, por citar un ejemplo de distinto corte, el espacio también tiene un claro valor simbólico. A lo largo de la historia, las calles tienen un cariz positivo en la vida de Felipe Centeno, pues es en ellas donde se libera del espacio opresor de la casa de los Polo, en la que sirve y no recibe un trato demasiado agradable.

Sin embargo, a pesar de este claro valor simbólico que Galdós otorga al espacio, que es del que se ha ocupado la crítica especializada de manera más frecuente, en este artículo hemos decidido centrarnos en la forma misma que el autor tiene de tratar el espacio desde un punto de vista textual, pues también tiene un valor estilístico que ha pasado desapercibido para la crítica especializada —o al menos no ha sido abordado de forma sistemática - Es cierto que en algunos estudios se abordan aspectos como los deícticos espaciales (cf. Arroyo Díez, 2011: 43 y ss.), pero no existen trabajos en los que se traten, de forma metódica, los hábitos sobre los que Galdós construye su magistral dominio del espacio en la creación de sus universos ficticios. Las construcciones que aquí se analizan revelan patrones formales y funcionales que ayudarán a entender mejor el estilo del autor canario. El objetivo del artículo es, como se ha indicado, localizar el uso sistemático de estos patrones y poder ahondar en sus funciones. Debido a la vasta producción de Galdós, con una extensión de casi seis millones y medio de palabras, esta tarea solo resulta posible gracias a un enfoque de corpus como el que se explica a continuación.

\section{CORPUS DE ESTUDIO Y METODOLOGÍA EMPLEADA}

El enfoque empleado se basa en el procesamiento del corpus de la producción narrativa de Galdós - y del corpus referencia formado 
por novela realista que hemos utilizado a modo de comparación- con WordSmith Tools 7 (Scott, 2016), un software de concordancias que permite identificar los clusters que contienen referencias espaciales que aquí se analizan. Con respecto a los textos que forman el corpus de novelas de Galdós (en adelante, CorBPG) y el corpus de novela realista (en adelante, CorXIX), estos se han recopilado de la Biblioteca Virtual Miguel de Cervantes. Este portal, que tiene como objetivo la difusión de la cultura hispánica en general, cuenta con alrededor de doscientos mil registros bibliográficos, de los cuales sesenta mil son libros. La calidad de la versión digitalizada de los textos, fundamental en los estudios de corpus como el que aquí se realiza, está fuera de toda duda, pues la Biblioteca cuenta con un consejo científico que avala el rigor de los materiales alojados en el repositorio. En el caso de Galdós, toda su producción literaria se encuentra digitalizada. Para llevar a cabo este análisis, se ha seleccionado su obra narrativa, con una extensión de más de seis millones de palabras, como se muestra en la tabla 1. La narrativa breve no ha sido incluida en el corpus, por no tratarse de novelas propiamente dichas. Tampoco ha sido incluida su producción teatral, por no pertenecer al género narrativo.

\begin{tabular}{|l|l|l|}
\hline Novela & Año & Palabras \\
\hline Episodios Nacionales. Primera serie & 1873 & 51.453 \\
\hline Trafalgar & 1873 & 139.656 \\
\hline La Corte de Carlos IV & 1873 & 136.403 \\
\hline El 19 de Marzo y el 2 de Mayo & 1873 & 63.819 \\
\hline Bailén & 1874 & 153.971 \\
\hline Napoleón en Chamartín & 1874 & 133.010 \\
\hline Zaragoza & 1874 & 58.453 \\
\hline Gerona & 1874 & 76.724 \\
\hline Cádiz & 1874 & 63.757 \\
\hline Juan Martín El Empecinado & 1875 & 90.064 \\
\hline La Batalla de los Arapiles & &
\end{tabular}




\begin{tabular}{|l|l|l|}
\hline Episodios Nacionales. Segunda serie & 1875 & 61.043 \\
\hline El equipaje del Rey José & 1875 & 51.324 \\
\hline Memorias de un cortesano de 1815 & 1876 & 65.663 \\
\hline La Segunda Casaca & 1876 & 64.034 \\
\hline El Grande Oriente & 1876 & 51.624 \\
\hline 7 de Julio & 1877 & 56.331 \\
\hline Los Cien Mil Hijos de San Luis & 1877 & 67.105 \\
\hline El Terror de 1824 & 1878 & 66.867 \\
\hline Un voluntario realista & 1879 & 77.113 \\
\hline Los Apostólicos & 1879 & 79.761 \\
\hline Un faccioso más y algunos frailes menos & \multicolumn{2}{|l|}{} \\
\hline Episodios Nacionales. Tercera serie & 1898 & 71.013 \\
\hline Zumalacárregui & 1898 & 83.240 \\
\hline Mendizábal & 1898 & 80.346 \\
\hline De Oñate a La Granja & 1899 & 93.695 \\
\hline Luchana & 1899 & 73.299 \\
\hline La campaña del Maestrazgo & 1899 & 67.799 \\
\hline La estafeta romántica & 1899 & 77.776 \\
\hline Vergara & 1900 & 61.666 \\
\hline Montes de Oca & 1900 & 75.899 \\
\hline Los Ayacuchos & 1900 & 74.812 \\
\hline Bodas reales & \multicolumn{2}{|l|}{} \\
\hline Episodios Nacionales. Cuarta serie & 1902 & 73.726 \\
\hline Las tormentas del 48 & 1902 & 82.797 \\
\hline Narváez & 1903 & 72.494 \\
\hline Los duendes de la camarilla & $1903-04$ & 77.393 \\
\hline La Revolución de Julio & 1904 & 78.986 \\
\hline O’Donnell & $1904-05$ & 78.507 \\
\hline Aita Tettauen & 1905 & 72.412 \\
\hline Carlos VI en la Rápita & 1906 & 70.956 \\
\hline La vuelta al mundo en la Numancia & \multicolumn{2}{l}{} \\
\hline
\end{tabular}




\begin{tabular}{|l|l|l|}
\hline Prim & 1906 & 79.099 \\
\hline La de los tristes destinos & 1907 & 87.885 \\
\hline Episodios Nacionales. Quinta serie & $1907-08$ & 78.784 \\
\hline España sin Rey & 1909 & 76.574 \\
\hline España trágica & 1910 & 73.434 \\
\hline Amadeo I & 1911 & 68.228 \\
\hline La Primera República & 1911 & 68.285 \\
\hline De Cartago a Sagunto & 1912 & 68.306 \\
\hline Cánovas & \multicolumn{2}{|l|}{} \\
\hline Novelas serie primera época & 1870 & 125.722 \\
\hline La Fontana de Oro & 1870 & 48.343 \\
\hline La sombra; Celín; Tropiquillos; Theros & 1871 & 119.174 \\
\hline El audaz: historia de un radical de antaño & 1876 & 65.486 \\
\hline Doña Perfecta: novela original & $1876-77$ & 127.697 \\
\hline Gloria & 1878 & 51.234 \\
\hline Marianela & 1878 & 145.720 \\
\hline La familia de León Roch & \multicolumn{2}{|l|}{} \\
\hline Novelas serie contemporánea & 1881 & 141.130 \\
\hline La desheredada & 1882 & 90.703 \\
\hline El amigo Manso & 1883 & 113.949 \\
\hline El doctor Centeno & 1884 & 83.370 \\
\hline Tormento & 1884 & 71.862 \\
\hline La de Bringas & $1884-85$ & 172.610 \\
\hline Lo prohibido & $1886-87$ & 379.607 \\
\hline Fortunata y Jacinta & 1888 & 97.628 \\
\hline Miau & 1889 & 75.503 \\
\hline La incógnita & 1889 & 76.091 \\
\hline Realidad: novela en cinco jornadas & 62.658 \\
\hline $\begin{array}{l}\text { Torquemada en la hoguera; El artículo de fondo; La mula y el } \\
\text { buey; La pluma en el viento; La conjuración de las palabras; } ; \\
\text { Un tribunal literario; La princesa y el granuja; Junio }\end{array}$ & \\
\hline & 1889 & \\
\hline
\end{tabular}




\begin{tabular}{|l|l|l|}
\hline Torquemada en la Cruz & 1893 & 58.302 \\
\hline Torquemada en el Purgatorio & 1894 & 67.625 \\
\hline Torquemada y San Pedro & 1895 & 61.367 \\
\hline Ángel Guerra & $1890-91$ & 249.534 \\
\hline Tristana & 1892 & 52.808 \\
\hline La loca de la casa & 1892 & 36.527 \\
\hline Nazarín & 1895 & 65.019 \\
\hline Halma & 1895 & 76.162 \\
\hline Misericordia & 1897 & 84.131 \\
\hline El abuelo & 1897 & 68.161 \\
\hline Total palabras & & 6.443 .709 \\
\hline
\end{tabular}

Tabla 1. Novelas incluidas en CorBPG

El corpus de referencia de novela realista, por su parte, está compuesto por ochenta novelas de ocho autores diferentes: Pedro Antonio de Alarcón, Vicente Blasco Ibáñez, Leopoldo Alas “Clarín”, Luis Coloma, Armando Palacio Valdés, Emilia Pardo Bazán, José María de Pereda y Juan Valera. Las ochenta novelas tienen una extensión similar a CorBPG, con 6.188.478 palabras. Los títulos que conforman CorXIX se muestran en la tabla $2^{1}$. Este corpus se ha utilizado por una cuestión de comparación, con el fin de calibrar la relevancia de los resultados obtenidos en la producción de Galdós.

${ }^{1}$ El año que se muestra en la tabla se corresponde con la edición de la Biblioteca Virtual Miguel de Cervantes, que no es necesariamente el mismo que el año original de publicación de la obra. 


\begin{tabular}{|c|c|c|c|}
\hline Autor & Novela & Año & Palabras \\
\hline \multirow{4}{*}{ Alarcón } & El final de Norma & 1855 & 41.892 \\
\hline & El sombrero de tres picos & 1874 & 24.909 \\
\hline & El escándalo & 1875 & 78.583 \\
\hline & El Capitán Veneno & 1881 & 40.655 \\
\hline \multirow{12}{*}{ Blasco Ibáñez } & Arroz y tartana & 1894 & 91.328 \\
\hline & La barraca & 1898 & 54.575 \\
\hline & Entre naranjos & 1900 & 80.873 \\
\hline & Cañas y barro & 1902 & 4.913 \\
\hline & La catedral & 1903 & 98.430 \\
\hline & El intruso & 1904 & 96.969 \\
\hline & La horda & 1905 & 101.600 \\
\hline & Los argonautas. & 1914 & 205.215 \\
\hline & Los cuatro jinetes del Apocalipsis & 1916 & 132.439 \\
\hline & Los enemigos de la mujer & 1919 & 163.461 \\
\hline & El paraiso de las mujeres & 1922 & 90.540 \\
\hline & El fantasma de las alas de oro & 1930 & 70.788 \\
\hline \multirow{2}{*}{ Clarín } & La Regenta & $1884-85$ & 309.796 \\
\hline & Su único hijo & 1890 & 89.969 \\
\hline \multirow{7}{*}{ Coloma } & La almohadita del niño Jesús & 1887 & 21.753 \\
\hline & Pequeñeces & $1890-91$ & 20.246 \\
\hline & Cuentos para niños & 1890 & 17.095 \\
\hline & La reina mártir & 1898 & 77.077 \\
\hline & Jeromin & 1902 & 146.177 \\
\hline & Colección de lecturas recreativas & 1902 & 216.807 \\
\hline & Por un piojo...: cuadro de costumbres & 1912 & 26.915 \\
\hline \multirow{4}{*}{ Palacio Valdés } & El idilio de un enfermo & 1884 & 58.113 \\
\hline & La aldea perdida & 1903 & 107.153 \\
\hline & La novela de un novelista & 1921 & 103.561 \\
\hline & Sinfonía pastoral & 1931 & 107.154 \\
\hline
\end{tabular}




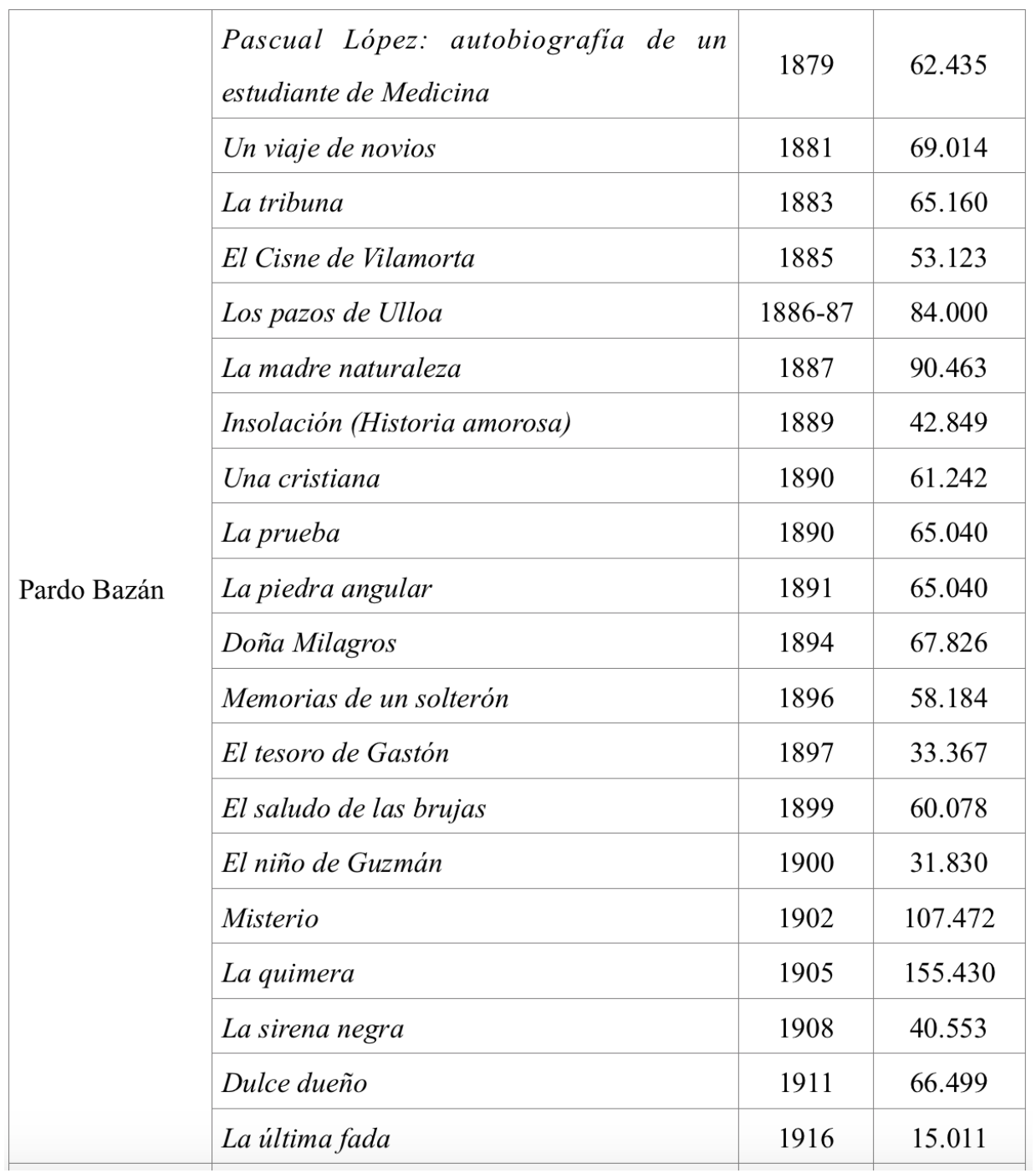




\begin{tabular}{|c|c|c|c|}
\hline \multirow{19}{*}{ Pereda } & Escenas montañesas & 1864 & 89.339 \\
\hline & Tipos y paisajes & 1871 & 100.753 \\
\hline & Don Gonzalo González de la Gonzalera & 1878 & 88.297 \\
\hline & El buey suelto... & 1878 & 72.537 \\
\hline & De tal palo tal astilla & 1880 & 85.974 \\
\hline & El sabor de la tierruca & 1882 & 81.756 \\
\hline & Pedro Sánchez & 1883 & 110.193 \\
\hline & Sotileza & 1885 & 117.441 \\
\hline & Esbozos y rasguños & 1888 & 70.323 \\
\hline & La Montálvez & 1888 & 106.115 \\
\hline & La mujer del César & 1888 & 25.179 \\
\hline & Los hombres de pro & 1888 & 45.060 \\
\hline & Tipos trashumantes & 1888 & 29.631 \\
\hline & Nubes de estio & 1891 & 121.738 \\
\hline & Al primer vuelo: Idilio vulgar. & 1891 & 100.914 \\
\hline & Oros son triunfos & 1894 & 27.233 \\
\hline & Peñas arriba & 1895 & 134.976 \\
\hline & Pachín González & 1896 & 60.309 \\
\hline & Escritos de juventud & 1954 & 82.726 \\
\hline \multirow{10}{*}{ Valera } & Pepita Jiménez & 1874 & 56.575 \\
\hline & Las ilusiones del doctor Faustino & 1875 & 119.890 \\
\hline & Pasarse de listo & 1878 & 51.851 \\
\hline & Morsamor & 1899 & 84.574 \\
\hline & Juanita la Larga & 1895 & 73.329 \\
\hline & Genio y figura & 1897 & 53.358 \\
\hline & Elisa la malagueña & 1964 & 11.844 \\
\hline & Leyendas del antiguo oriente & 1964 & 35.652 \\
\hline & Mariquita y Antonio & 1964 & 50.824 \\
\hline & Doña Luz & 1879 & 59.517 \\
\hline Valera & El comendador Mendoza & 1876 & 63.355 \\
\hline
\end{tabular}

Tabla 2. Novelas incluidas en CorXIX 
Para analizar el espacio en la producción de Galdós, se han generado clusters tanto en CorBPG como en CorXIX y se han ordenado según su frecuencia en ambos corpus. Los clusters son grupos de palabras de una longitud determinada generados automáticamente por un software de concordancias — en nuestro caso, WordSmith Tools - . El hecho de que no se seleccionen de forma premeditada refuerza, sin duda, su relevancia estilística. Para obtener una relación de ejemplos lo más significativa posible, hemos seleccionado aquellos clusters con una longitud mínima de cinco palabras y una frecuencia de al menos cinco apariciones en cada corpus $^{2}$. Los resultados obtenidos se muestran en el siguiente apartado.

\section{RESULTADOS}

Por una cuestión de representatividad, nos hemos centrado en los clusters más numerosos identificados en cada corpus. En concreto, hemos seleccionado los primeros veinticinco de CorBPG y CorXIX. Estos se muestran en la tabla 3.

\begin{tabular}{|l|l|l|l|l|}
\hline \multicolumn{2}{|c|}{ CorBPG } & \multicolumn{2}{c|}{ CorXIX } & \\
\hline & Cluster & Frec. & Cluster & Frec. \\
\hline $\mathbf{1}$ & A UN LADO Y OTRO & 126 & $\begin{array}{l}\text { LA NOVELA DE UN } \\
\text { NOVELISTA }\end{array}$ & 157 \\
\hline $\mathbf{2}$ & DE LA CALLE DE LA & 126 & A LA PUERTA DE LA & 135 \\
\hline $\mathbf{3}$ & EN LA CALLE DE LA & 98 & DE UN MOMENTO A OTRO & 79 \\
\hline $\mathbf{4}$ & EN LA PUERTA DE LA & 88 & DE UN LADO A OTRO & 77 \\
\hline $\mathbf{5}$ & EN LA CUENTA DE QUE & 81 & AL FIN Y AL CABO & 73 \\
\hline $\mathbf{6}$ & CASA DE LA CALLE DE & 74 & AL PIE DE LA LETRA & 71 \\
\hline $\mathbf{7}$ & EN MEDIO DE LA CALLE & 70 & EN EL FONDO DE SU & 68 \\
\hline
\end{tabular}

${ }^{2}$ Para una información detallada de qué es un cluster y cómo se genera utilizando WordSmith Tools, véase Scott (2013). 


\begin{tabular}{|l|l|l|l|l|}
\hline $\mathbf{8}$ & EN EL MOMENTO EN QUE & 69 & DE DON JUAN DE AUSTRIA & 67 \\
\hline $\mathbf{9}$ & A LA PUERTA DE LA & 65 & LA MAYOR PARTE DE LOS & 62 \\
\hline $\mathbf{1 0}$ & EN LA PUERTA DEL SOL & 65 & A UN LADO Y A & 57 \\
\hline $\mathbf{1 1}$ & LA PUERTA DE LA CASA & 61 & NI MÁS NI MENOS QUE & 57 \\
\hline $\mathbf{1 2}$ & DE UN LADO PARA OTRO & 60 & A UN LADO Y A OTRO & 56 \\
\hline $\mathbf{1 3}$ & LA ESQUINA DE LA CALLE & 59 & EN LA CUENTA DE QUE & 56 \\
\hline $\mathbf{1 4}$ & LAS MANOS A LA CABEZA & 59 & UN LADO Y A OTRO & 56 \\
\hline $\mathbf{1 5}$ & YO NO SÉ LO QUE & 56 & A LA LUZ DE LA & 55 \\
\hline $\mathbf{1 6}$ & POR LA CALLE DE LA & 55 & LAS MANOS EN LS & 55 \\
\hline $\mathbf{1 7}$ & NO HAY MÁS REMEDIO & 53 & EN EL FONDO DE LA & 52 \\
\hline $\mathbf{1 8}$ & NO SÉ LO QUE ME & 53 & EN EL MOMENTO EN QUE & 49 \\
\hline $\mathbf{1 9}$ & DE UNA PARTE A OTRA & 52 & LAS TRES DE LA TARDE & 49 \\
\hline $\mathbf{2 0}$ & DE LA CALLE DE SAN & 51 & LE DIGO A USTED QUE & 49 \\
\hline $\mathbf{2 1}$ & NO ES MÁS QUE UN & 51 & LA PALMA DE LA MANO & 48 \\
\hline $\mathbf{2 2}$ & $\begin{array}{l}\text { NO HABÍA MÁS REMEDIO } \\
\text { QUE }\end{array}$ & 51 & LA PUERTA DE LA CALLE & 47 \\
\hline $\mathbf{2 3}$ & AL CAER DE LA TARDE & 49 & A LA LUZ DEL SOL & 46 \\
\hline $\mathbf{2 4}$ & A LA CALLE DE LA & 48 & LA MAYOR PARTE DE LAS & 46 \\
\hline $\mathbf{2 5}$ & DE LA NOCHE A LA & 48 & A DON JUAN DE AUSTRIA & 45 \\
\hline
\end{tabular}

Tabla 3. Clusters identificados en CorBPG y CorXIX

Como se puede advertir, la mayoría de los ejemplos localizados en CorBPG contienen referencias al espacio. En concreto, quince de los veinticinco clusters más numerosos identificados en la obra narrativa de Galdós tienen que ver con cuestiones relacionadas con el espacio ( $a$ un lado y otro, de la calle de la, en la calle de la o en la puerta de la, por mencionar los cuatro primeros, todos con referencias espaciales). Esta es, sin duda, una clara señal de la importancia del espacio, apuntada ya por la crítica especializada. El hecho de que el procesamiento del corpus galdosiano apunte en esta dirección resulta interesante, pues, como señala Stubbs, "even if quantification only confirms what we already know, this is no bad thing [...], since this gives confidence that the method can be 
relied on" (Stubbs, 2005: 6). Y en este caso, no solo nos vale para "confirm findings from many years of traditional study" (Stubbs, 2005: 6), sino para calibrarlos con exactitud: el espacio se presenta como un elemento fundamental en el estilo galdosiano, con quince de los veinticinco clusters más numerosos relacionados con este aspecto. Esta relevancia se refuerza, además, si observamos el conjunto de clusters identificados en CorXIX. Como se puede observar, solo seis de los veinticinco ejemplos localizados hacen referencia a cuestiones relacionadas con el espacio (a la puerta de la, de un lado a otro, a un lado y a, a un lado y a otro, un lado y a otro y la puerta de la calle). Este hecho no solo justifica, sino que parece demandar un estudio detenido como el que aquí se presenta, en el que se aborden los mecanismos empleados por Galdós para definir el espacio en sus novelas.

\section{ANÁLISIS}

Para llevar a cabo el análisis hemos realizado concordancias de los quince clusters localizados en CorBPG que contienen referencias espaciales, identificando todos los ejemplos de cada construcción en listas separadas. Esto permite una lectura vertical de los ejemplos (Tognini-Bonelli, 2001: 18), que hace posible, a su vez, identificar patrones recurrentes a lo largo de la producción narrativa de Galdós. Para ilustrar los hallazgos más significativos de nuestro estudio, hemos dividido el apartado analítico en dos grandes bloques. En primer lugar, hemos clasificado los clusters en bloques separados según el tipo de referencia espacial que contienen y hemos analizado las funciones principales de cada uno estos bloques (apartado 5.1). En segundo lugar, nos hemos centrado en cómo Galdós recurre a estas construcciones con referencias espaciales para transmitir movimiento, lo que servirá para reforzar su consabido dominio del espacio a través de un análisis de aspectos que hasta ahora no habían sido descritos —o al menos analizados de forma metódica (apartado 5.2) - 


\subsection{Clasificación}

Los quince clusters identificados en CorBPG se pueden clasificar en tres grandes grupos: los que contienen la palabra puerta, los que contienen la palabra calle y el resto, que contienen dos referencias espaciales ( $a$ un lado y otro, de un lado para otro y de una parte a otra). Que pueda realizarse una clasificación tan clara no parece casual, sino que más bien es el resultado de la existencia de patrones en los que Galdós se apoya para definir el espacio visual y construir su universo ficticio. De hecho, si observamos el uso que Galdós hace de los distintos clusters que conforman cada uno de los bloques, advertimos patrones que refuerzan esta tesis, como se detalla a continuación.

\subsubsection{Las calles}

El bloque de clusters que contienen la palabra calle es el más variado, con ocho ejemplos (de la calle de la, en la calle de la, casa de la calle de, en medio de la calle, la esquina de la calle, por la calle de la, de la calle de San y a la calle de la). La aparición recurrente de las calles entre los clusters más numerosos refleja la importancia de este elemento en la obra de Galdós. Este hecho se refuerza si tenemos en cuenta la frecuencia relativa de calle en CorBPG y CorXIX, siendo de $0,07 \%$ en el primero (4674 apariciones totales) y $0,03 \%$ en el segundo (2164 apariciones totales). En el género novelesco, las calles son una parte importante del paisaje urbano en la época realista (González, 1998: 32). En el caso concreto de Galdós, el autor recurre habitualmente a estos espacios para situar la acción presentada en un espacio real, de manera que logra un alto grado de verosimilitud en el marco del antedicho paisaje urbano en el que transcurren la mayoría de sus historias. Además de esta sensación de realismo a la que se suele referir la crítica tradicional, la identificación de los clusters que contienen la palabra calle permite ahondar en el uso que Galdós hace de este elemento desde un punto de vista textual y descubrir 
patrones que se repiten a lo largo de la producción del autor canario. E1 uso más habitual es el de posicionar lugares en los que transcurre la acción mediante su enclave en una vía determinada. Pisos, cuadras, cuartitos, colegios, prisiones, farmacias, tiendas, auditorios, cacharrerías, buñolerías, comercios, pollerías, clubes y sobre todo casas - casa de la calle es el sexto cluster más habitual en el corpus galdosiano, con setenta y cuatro ejemplos - son localizaciones a las que se suele hacer referencia por su situación en una calle concreta, como se puede observar en el siguiente ejemplo extraído de La desheredada, en el que se advierten sendas referencias a las calles en las que se sitúan un comercio y una cacharrería.

Mi comercio de la calle del Pez se hizo agua una noche para sacarle de la cárcel, cuando aquel feo negocio de los billetes de lotería. La cacharrería de la calle de la Torrecilla se resquebrajó después, y pieza por pieza se la fueron tragando el médico y el boticario, cuando cayó Francisca en la cama con la enfermedad que se la llevó (Pérez Galdós, 1909: 48).

Este gusto por incidir en la situación de determinados lugares en la ciudad no se advierte en CorXIX —o al menos no con la misma intensidad-. Se trata más bien de un recurso galdosiano, que encaja con su dominio del espacio visual y con el que confiere sensación de realismo a sus universos ficticios.

Un uso análogo se observa con los personajes, lo que refuerza este hábito estilístico. Así, los personajes se identifican frecuentemente por su localización. El carbonero de la calle de la Esperancilla (Tormento), las monjitas de la calle de San Leonardo (Amadeo I), el prestamista de la calle de San Blas (Torquemada en la hoguera) o el guarnicionero en la calle de la Zapatería de Viejo (El 19 de Marzo y el 2 de Mayo) son solo algunos ejemplos. En ocasiones se trata de referencias que sirven para construir el universo ficticio, pues se ofrecen detalles que van más allá del personaje. Esto es lo que ocurre en el primero de los ejemplos siguientes, donde el 
guarnicionero de El 19 de Marzo y el 2 de Mayo se nos describe como un personaje "instalado en la calle de la Zapatería de Viejo" y además se nos ofrecen datos más concretos de la localización ("muy contigua a la de la Sal"). De forma más frecuente, las calles sirven como referencia de identificación de los personajes, es decir, las calles actúan como una suerte de recurso de caracterización. Esto es lo que ocurre con las monjas de la calle de San Leonardo en el segundo de los ejemplos, extraído de Amadeo I. Además de como un recurso de caracterización, referencias como la de "la calle de San Leonardo" para identificar a las monjas nos ayudan a su vez a formar el universo ficticio que el autor nos plantea, pues los personajes aparecen frecuentemente presentados como pertenecientes a un punto concreto del mapa de la historia.

Por fortuna, yo conocía un maestro guarnicionero instalado en la calle de la Zapatería de Viejo, muy contigua a la de la Sal, y resolvi dirigirme a él para pedir informes del Sr. Requejo (Pérez Galdós, 1875a: 130).

Enterose de ello la Marquesa de Navalcarazo, y queriendo apartar a la pobre niña de todo influjo maléfico, obligó a la madre a ponerla bajo la guardia y custodia de unas monjitas de la calle de San Leonardo (Pérez Galdós, 1910: 140).

En suma, además de para definir la escena, Galdós emplea las calles de modo referencial, con las que establece conexiones entre lugares y también personajes con enclaves en el marco de la ciudad. Este uso tan particular - y tan habitual, pues es el que se advierte en los usos del término calle en las construcciones más comunes en las que se emplea el sustantivo en la producción del autor- supone un hábito estilístico representativo del que se vale para construir su universo ficticio. Este hábito sirve para reforzar el magistral uso que Galdós hace de las calles descrito por la crítica, en el que estas, como centro de la vida urbana, 
desempeñan un papel protagonista a través de minuciosas descripciones y su papel protagonista como enclave donde se desarrollan los hechos (Arroyo Díez, 2011: 103).

\subsubsection{Las puertas}

Por su parte, los clusters que contienen la palabra puerta (en la puerta de la, a la puerta de la, en la puerta del Sol y en la puerta de la casa) son ante todo ejemplos de punto de emplazamiento. Dicho de otro modo, en el universo ficticio galdosiano las puertas suelen actuar como la referencia en la que se sitúan los personajes y en torno a las que se construye la escena que se nos presenta. Al contrario de lo que ocurre con las calles, la frecuencia normalizada de puerta en la obra del canario (3486 ejemplos, lo que constituye un $0,05 \%$ del corpus) no es mayor que en la novela realista en general (3253 ejemplos en CorXIX, lo que constituye también un $0,05 \%$ del corpus). Sin embargo, el uso tan particular detectado en CorBPG sí que revela un rasgo típico del estilo de Galdós. En concreto, a lo largo de sus novelas es frecuente toparse con referencias a la puerta de la casa, la puerta de la cocina, la puerta de la alcoba, la puerta de la armería, la puerta de la ratonera, la puerta de la iglesia, la puerta de la carnicería, la puerta de la botica, la puerta de la comandancia, etc. como matrices sobre las que se construye la escena. En los fragmentos que incluimos a continuación se muestran dos ejemplos. En el primero, perteneciente a Fortunata y Jacinta, Galdós utiliza la puerta de la alcoba de Doña Lupe como punto de referencia para explicar la posición de Fortunata. Sobre la puerta se construye la foto que recibimos como lectores. Algo parecido ocurre en el segundo caso, extraído de O'Donnell. A pesar de ofrecérsenos una descripción tanto física como introspectiva de Mita, esta se realiza nuevamente tomando como referencia su posición: sentada en la puerta de la casa. Estos usos encajan dentro del planteamiento que Galdós hace del espacio como recurso con el que lograr un entorno físico realista sobre el que desarrollar la acción. 
Fortunata le examinaba atentamente, sentada lejos del grupo principal, en una silla próxima a la puerta de la alcoba de doña Lupe. Él no se sentó [...] (Pérez Galdós, 1887: 59).

Mita, sentada en la puerta de la casa, expresaba con su inmovilidad, el codo en la rodilla, la cara recostada en la palma de la mano, el aburrimiento de una larga espera (Pérez Galdós, 1904: 331).

Otro uso más específico, y que no ha sido abordado por la crítica especializada, es el que marca la puerta como finalización de un hecho. En los pasajes que aparecen a continuación, pertenecientes a Ángel Guerra y Miau respectivamente, se muestran dos usos del cluster la puerta de la casa en los que la puerta se presenta no solo como el punto donde se sitúan los personajes, sino como referencia sobre la que se construye el final del episodio que tiene lugar. En ambos ejemplos, la puerta es donde se detienen los personajes para despedirse. Este uso tan concreto se puede añadir al hábito galdosiano de situar la casa donde vive un personaje "de prolegómeno de una escena que va a venir y de prólogo de otros pasajes importantes de la narración” (Arroyo Díez, 2011: 102). No es extraño, como ocurre en los siguientes ejemplos, que, además de resultar un prolegómeno, las referencias espaciales —en nuestro caso la puerta de la casa- sean también un elemento que marca el punto final de la escena.

Nada contestó Guerra a estas sesudas palabras. Detuviéronse a la puerta de la casa del arquitecto, y se despidieron apretándose cariñosamente las manos, y deseándose una buena noche [...] (Pérez Galdós, 1891: 321).

En esto llegaron a la puerta de la casa mortuoria, donde Quintina, después de besuquearle otra vez refregándole la cara, le dejó en 
compañia de los demás chicos, que ya estaban allí, más de lo que permitían las tristes circunstancias (Pérez Galdós, 1888: 277).

\subsubsection{Dos referencias espaciales}

Por último, aunque el bloque de los clusters con dos referencias espaciales es el menos variado (solo tres ejemplos: a un lado y otro, de un lado para otro y de una parte a otra), en él se advierten dos patrones estilísticamente relevantes desde el punto de vista de la construcción del universo ficticio galdosiano. El primero tiene que ver, como es fácil imaginar, con el movimiento. Al existir dos referencias, estas se erigen habitualmente en puntos de salida y llegada de un movimiento, expresado por el verbo - de carácter cinético- con el que se asocian (andar, correr, ir, mover, pasear, transportar, etc.). A continuación mostramos dos ejemplos de corte similar, en los que la construcción, además de ayudar a configurar el movimiento en la escena, contribuye a reforzar el carácter ansioso de los personajes. En el primer caso, perteneciente a Los apostólicos, de un lado para otro se asocia con el verbo moverse y nos presenta la intranquilidad de Doña Carlota a través de sus movimientos por la casa. En el segundo ejemplo, por su parte, el cluster se asocia con el verbo ir, y desempeña una función similar en la proyección de la inquietud de Amparo en Tormento. Esta es una de las formas que Galdós emplea para transmitir movimiento utilizando referencias espaciales, junto con las que se explican en el siguiente apartado.

En pie y moviéndose sin cesar de un lado para otro, altiva, nerviosa, respirando fuerte, Doña Carlota parecía que imaginaba crueldades y violencias impropias de mujer y de princesa (Pérez Galdós, 1879: 374).

Iba de una parte a otra de la casa con morbosa inquietud; y en ocasiones veía los objetos del revés, invertidos. Hasta el retrato 
de su padre tenía la cabeza hacia abajo (Pérez Galdós, 1874: 115).

Cuando no tienen que ver con el movimiento, los clusters con dos referencias espaciales suelen relacionarse con la definición del espacio en el que se desarrolla la escena. Este parece ser un estilema galdosiano en la construcción del universo ficticio que el autor nos plantea, sobre todo por el uso tan peculiar que hace de él. En concreto, la particularidad de este uso se encuentra en la sorprendente frecuencia con la que Galdós utiliza este cluster con la visión de los personajes como matriz desde la que construir la escena. En la novela realista en general, muchos personajes tienen la función de mirar, y esta mirada es el recurso empleado por el narrador para insertar descripciones (Zubiaurre, 2000: 24). En el caso de Galdós, como se ha encargado de demostrar la crítica, el espacio se presenta con frecuencia a través del punto de vista del personaje (Arroyo Díez, 2011: 47). En muchos de estos casos se observa el uso de clusters con dos referencias espaciales. Con esta estrategia, Galdós focaliza el relato en la mirada de los personajes, lo que hace que, como lectores, presenciemos la escena a través de sus miradas y construyamos la escena en nuestra imaginación. Los casos más claros se advierten en los usos de a un lado $y$ otro. En el siguiente fragmento perteneciente a Misericordia mostramos un ejemplo de este cluster, en el que la mirada sirve como mecanismo de engarce de la descripción en el texto narrativo. Como se puede advertir, Galdós se sirve de la mirada a un lado y otro de Benina para ofrecer, con todo lujo de detalles, la escena que pretende mostrarnos cuando esta sale de casa, desde una curva en forma de zigzag que conduce a la estación de las pulgas hasta "un arroyo de aguas de alcantarilla, negras como tinta, baja por un cauce abierto en los taludes, y salvando el camino por una atarjea, corre a fecundar las huertas antes de verterse en el río". Todo depende de la mirada a ambos lados del personaje, responsable de la información que recibimos y la que guía nuestra interpretación, como a veces suele ocurrir en el género novelesco (cf. Garrido, 1993: 220). 
Dando las gracias a la esmirriada, salió Benina, y se fue por toda la calle adelante, atisbando a un lado y otro. Esperaba distinguir en alguno de aquellos calvos oteros la figura del marroqui tomando el sol o entregado a sus melancolías. Pasadas las casas de Ulpiano, no se ven a la derecha más que taludes áridos y pedregosos, vertederos de escombros, escorias y arena. Como a cien metros de la explanada hay una curva o más bien zig-zag, que conduce a la estación de las Pulgas, la cual se reconoce desde abajo por la mancha de carbón en el suelo, las empalizadas de cerramiento de vía, y algo que humea y bulle por encima de todo esto. Junto a la estación, al lado de Oriente, un arroyo de aguas de alcantarilla, negras como tinta, baja por un cauce abierto en los taludes, y salvando el camino por una atarjea, corre a fecundar las huertas antes de verterse en el rio (Pérez Galdós, 1897: 255).

Estos usos tan específicos, junto a los identificados en los clusters que contienen referencias a las calles y las puertas, revelan hábitos estilísticos en la narrativa de Galdós en lo que a la construcción de sus universos ficticios a través del tratamiento de aspectos espaciales se refiere. Un estudio de corpus permite no solo identificarlos sino analizarlos de forma metódica, lo que refuerza algunos de los presupuestos estilísticos sobre los que se cimienta la opinión de la crítica especializada y revela también algunos usos que hasta ahora habían pasado desapercibidos en la exégesis del autor canario.

\subsection{Patrones funcionales}

Además de estos usos independientes identificados al clasificar los clusters en tres categorías - usos que podríamos asociar al tratamiento que Galdós da a unidades concretas como las calles, las puertas o la 
combinación de referencias espaciales-, el conjunto de quince clusters identificados también puede analizarse de forma integral, lo que revela mecanismos estilísticos de los que Galdós se sirve para crear efectos que van más allá de la definición de la escena. Estas formas se abordan en este apartado. En concreto, nos centraremos en cómo Galdós utiliza las referencias espaciales para crear sensación de movimiento (apartado 5.2.1) y también para dotar de ritmo a la acción mediante la creación de impresión de simultaneidad entre lo que está ocurriendo en el plano discursivo y los aspectos que rodean a las conversaciones, marcados habitualmente por elementos que definen la escena (apartado 5.2.2).

\subsubsection{Creación de movimiento}

Además de los clusters con dos referencias espaciales analizados en 5.1.3, cabe destacar que los que contienen una sola referencia también son empleados por Galdós para crear sensación de movimiento. Como cabría esperar, y al igual que en el caso de los ejemplos que contienen dos referencias espaciales, la constelación léxica (Cantos-Gómez y Sánchez, 2001) de los clusters con una sola referencia espacial — la puerta o la calleempleados para transmitir acción está formada principalmente por verbos que indican movimiento. Por ejemplo, a la puerta de se coloca con verbos como entrar, ir o desplazarse. Sin embargo, lo verdaderamente interesante de estos clusters radica en su combinación con otras referencias espaciales. Esta combinación, junto al verbo de carácter cinético que describe la acción, refuerza la sensación de movimiento descrita por el narrador, como se muestra en el siguiente ejemplo de La Primera República, en el que, además de con el verbo recorrer, la puerta de la calle se combina con la capilla:

La ideal figura enlutada describió una suave curva para recorrer el camino desde la capilla a la puerta de la calle (Pérez Galdós, 1911: 120). 
Sin embargo, en la creación de sensación de movimiento por parte de Galdós también hay usos mucho más específicos en los que se advierte una sistematicidad que sugiere un estilema galdosiano a la hora de transmitir acción. Es aquí donde entra en juego su dominio del espacio visual y el movimiento, con el que "se prefigura el ritmo cinematográfico" (Arroyo Díez, 2011: 366) que caracteriza su estilo. Desde un punto de vista formal, cuando estos clusters se emplean para sugerir movimiento, aparecen en construcciones muy concretas que también ayudan a crear una suerte de movimiento en nuestra mente. En concreto, se trata de proposiciones subordinadas adverbiales de tiempo que aparecen precediendo a la proposición principal y que contienen una referencia espacial, como se describe en la figura 1.

Proposición subordinada adverbial de tiempo (cluster con referencia espacial)

proposición principal

Figura 1. Patrón empleado por Galdós para transmitir sensación de movimiento

Estas proposiciones ayudan a crear una sensación de transición de un punto (proposición subordinada) a otro (proposición principal). Es decir, existe una suerte de desplazamiento en el plano formal — de una proposición subordinada, dependiente de la principal, con la que se enlaza - que refleja el desplazamiento que percibimos en el contenido. No se trata de ejemplos aislados localizados con un cluster concreto, sino que aparecen tanto en ejemplos que contienen referencias a las calles como en ejemplos que contienen referencias a las puertas, como se puede observar en los siguientes fragmentos, pertenecientes a Miau y El 19 de Marzo y el 2 de Mayo respectivamente: 
Al entrar en la calle de la Puebla, iba ya Cadalsito tan fatigado que, para recobrar las fuerzas, se sentó en el escalón de una de las tres puertas con rejas que tiene en dicha calle el convento de Don Juan de Alarcón (Pérez Galdós, 1888: 294).

Cuando la puerta de la casa se abrió, precipitose la turba en lo interior, bramando de coraje (Pérez Galdós, 1875a: 90).

Como se puede advertir, la relación de subordinación refuerza el movimiento, pues la dependencia de la primera proposición sobre la segunda crea una suerte de transición que, en el caso oraciones coordinadas o yuxtapuestas, no existiría. Además, el hecho de que sea en la proposición subordinada donde aparece el cluster con la referencia espacial y que esta aparezca en posición inicial también fortalece la impresión de movimiento: como lectores, recibimos y procesamos la información relacionada con el espacio en primer lugar, lo que nos permite configurar la escena. Y desde ahí construimos el movimiento. Cabe destacar que estos casos aparecen tanto en fragmentos correspondientes a la voz del narrador - los ejemplos anteriores-, como en boca de los propios personajes, como se puede advertir en la siguiente intervención de Gervasio en Montes de Oca:

-Vas a saberlo. Ayer salíamos de almorzar en casa de Carriquiri, Narciso Ametller, Luis Sartorius y yo... Al volver la esquina de la calle de las Huertas, vimos a tu amiga salir de un coche con Federiquito Nieto, y entrar.. ¿sabes ya dónde? (Pérez Galdós, 1900: 178).

Tradicionalmente, la crítica se ha centrado en los personajes y su relación con el movimiento haciendo énfasis en que son los propios personajes "quienes se desplazan de un lugar a otro creando con su imaginación la visión que el lector ha de percibir de las calles de la ciudad" (Arroyo Díez 2011: 104). Ejemplos como el que acaba de mostrarse 
demuestran, además, que los personajes también imprimen movimiento a la narración cuando narran episodios de otros personajes a través de sus intervenciones. El hecho de que esta construcción tan concreta se dé tanto en fragmentos de la voz del narrador como en el discurso de los personajes no hace sino consolidar esta estrategia como un hábito estilístico galdosiano para configurar la impresión de movimiento.

\subsubsection{Simultaneidad de acciones}

Por último, las referencias espaciales también son empleadas de un modo muy concreto para crear impresión de simultaneidad entre el discurso de los personajes y sus movimientos. El patrón formal en el que se engarzan estos ejemplos suele ser siempre el mismo: se trata de casos de estilo directo en los que los clusters se colocan en la proposición proyectora, encontrándose esta en una posición media que interrumpe el discurso del personaje, como se muestra en el siguiente ejemplo de $E l$ equipaje del Rey José:

-Esto es horroroso - exclamó D. Fernando corriendo de un lado para otro en la oscura pieza-. Que nos fusilen... pero que no nos arrastren, ni nos destrocen, ni nos escupan, ni nos insulten... iPiedad, misericordia! (Pérez Galdós, 1875b: 208).

Estas proposiciones en las que se encuentran los clusters se denominan suspensiones. Una suspensión puede definirse como "a protracted interruption by the narrator of a character's speech" (Lambert, 1981: 6). Es un rasgo típico del estilo de Charles Dickens (Newsom, 2005: 556). La utilización sistemática de estas unidades por parte de Galdós parece ser un eco del novelista victoriano y la consabida influencia que ejerció en él (cf. Tambling). Desde un punto de vista estilístico, el uso de suspensiones que incluyen referencias espaciales en CorBPG parece tener que ver con la intención del autor de ofrecer un efecto de sincronía entre 
las palabras de los personajes y sus movimientos, pues la función de las suspensiones es la de crear "an impression of simultaneity between the speech and the contextual information described by the narrator, which in turn can suggest similarities to the simultaneous occurrence of speech and body language in real life" (Mahlberg, Smith y Preston, 2013: 40). La suspensión mostrada en el fragmento anterior, por ejemplo, transmite la sensación, en el acto de lectura, de que el movimiento de Don Fernando corriendo de un lado para otro tiene lugar al tiempo que articula sus palabras — que no es sino lo que ocurre en la historia-. Estos ejemplos encajan en la sensación de verosimilitud que Galdós es capaz de crear en la construcción de sus universos ficticios y que el lector llega a percibir como fragmentos de realidad (Gullón, 1980: 59).

En cuanto a su uso, las suspensiones en las que aparecen referencias espaciales se localizan principalmente en momentos de elevada carga emocional. Así lo demuestra el tono exclamativo que suele caracterizar el parlamento del personaje que las acompaña. En el caso del ejemplo anterior, es el verbo de habla (exclamó) el que nos lo indica. En otras ocasiones son las propias palabras del personaje las que aparecen entre signos de exclamación, como se muestra en los siguientes extractos de Vergara y 7 de Julio. Estas escenas se caracterizan por una alta intensidad que Galdós refuerza en el plano textual gracias al uso de las suspensiones, construcciones que transmiten sensación de simultaneidad entre las palabras cargadas de sentimentalismo que articulan los personajes y sus acciones.

—iAy, no, no, D. Eustaquio, por Jesús vivo! —exclamó ruborizada la señora, en la puerta de la cocina, secando un plato que acababa de fregar-. El pobre ingenio mio no merece tales honores [...] (Pérez Galdós, 1899: 222).

—;Gracias, gracias, mil gracias! — dijo galantemente el héroe saludando a un lado y otro-. Pero apartarse, apartarse, señoras 
(Pérez Galdós, 1876a: 216).

Sin embargo, estas suspensiones con referencias espaciales también se advierten en momentos menos emotivos. En tales casos, Galdós suele recurrir a los vocativos como estrategia para acomodar las suspensiones: el personaje que habla interpela a su interlocutor y es esta llamada la que se separa del resto del parlamento a través de la suspensión, como se puede observar en los siguientes ejemplos, pertenecientes a Napoleón en Chamartín y El doctor Centeno respectivamente. Este parece ser un uso ad hoc del vocativo, empleado para poder suspender la proposición proyectora que incluye la referencia espacial y crear la antedicha impresión de simultaneidad entre las palabras de los personajes y sus acciones.

— Gabriel —dijo deteniéndose en medio de la calle y asomando por el embozo de su capa un dedo con el cual ciceronianamente acentuaba sus palabras-, cuando yo lo digo, sabido me lo tengo [...] (Pérez Galdós, 1876b: 129).

-Rosa - dijo D. José, presentándose de improviso en la puerta de la cocina-. Vete a acostar al momento. Es muy tarde (Pérez Galdós, 1883: 273).

Por último, no podemos dejar de mencionar los casos en los que Galdós recurre a suspensiones con referencias espaciales cuando reproduce los pensamientos de sus personajes. Como se puede observar en los siguientes fragmentos de Fortunata y Jacinta y Vergara, se trata de la misma estrategia: estilo directo y el discurso (mental) del personaje interrumpido por las palabras del narrador (una suspensión), en las que se encuentra una referencia espacial. Aunque la crítica se ha ocupado tradicionalmente de analizar la interdependencia entre el movimiento físico y el proceso mental de los personajes, considerándosela un aspecto distintivo de la novela galdosiana (Arroyo Díez, 2011: 104), 
estos casos muestran un marcado patrón formal que arroja nueva luz sobre la interdependencia entre las acciones y los pensamientos de los personajes en el universo galdosiano. En concreto, el hecho de suspender la proposición que introduce los pensamientos y dividir estos en dos ayuda a crear una impresión de simultaneidad entre las acciones de los personajes y los pensamientos de los que nos informa el narrador, lo que contribuye a lograr un mayor grado de realismo en el plano textual.

-Pero ese tiro, ¿me lo doy o no me lo doy?... No tengo más remedio que dármelo - discurría entrando por la calle de la Magdalena - Por ninguna parte veo la solución [...] (Pérez Galdós, 1887: 283).

—Pero ¿qué reos son esos a quienes tengo yo que auxiliar? — me decía yo, vagando como un demente de una parte a otra con las manos en la cabeza - ¿Qué delito han cometido para que se les sacrifique inhumanamente? [...] (Pérez Galdós, 1899: 12).

Desde luego, resulta interesante —y lo que es más importante, estilísticamente significativo- que el uso de suspensiones para crear un efecto de sincronía entre el discurso de sus personajes (ya sea verbal o mental) y sus acciones esté caracterizado por la inclusión de referencias espaciales localizadas en los clusters más numerosos identificados en su producción narrativa. Este hecho no hace sino afianzar la relevancia estilística del espacio en la producción del autor, como se viene comentando a lo largo del artículo. Como se ha visto, la construcción de sus universos ficticios se basa, en gran medida, en el uso sistemático de determinadas construcciones. Además de para definir la escena (apartado 5.1), estas construcciones se emplean para transmitir sensación de movimiento e incluso sensación de simultaneidad entre acciones, como acabamos de ver. Los usos de estos clusters y sus funciones, algunas de ellas tradicionalmente desapercibidas en la exégesis del autor canario en lo que a su tratamiento 
del espacio se refiere, sirven para arrojar nueva luz sobre la maestría de Galdós en la construcción de sus universos ficticios, uno de los aspectos por los que goza de mayor reconocimiento.

\section{REFLEXIONES FINALES}

En este estudio hemos analizado el valor del espacio como uno de los ejes sobre los que se vertebra la creación del universo ficticio galdosiano. Para ello hemos utilizado un enfoque de estilística corpus. En concreto, el análisis se ha articulado en torno a clusters identificados gracias al procesamiento de los textos con un software de concordancias (WordSmith Tools), que ha revelado patrones tanto formales como funcionales que han pasado desapercibidos para la crítica tradicional. Esta es, precisamente, una de las mayores ventajas de los enfoques de corpus como el que aquí se ha empleado, pues permiten identificar segmentos de texto estilísticamente significativos en los que no reparamos como lectores (ni como críticos), si bien contribuyen igualmente a crear los efectos que percibimos en el acto de lectura. De forma más específica, la metodología empleada ha permitido localizar una serie de clusters que contienen referencias espaciales que, además de encontrarse entre las construcciones más empleadas por Galdós en sus novelas, son empleadas con un valor similar a lo largo de su producción. De hecho, ha sido posible clasificar los clusters identificados en tres grandes bloques: los que contienen la palabra calle, los que contienen la palabra puerta y los que contienen dos referencias espaciales. En ellos se advierten funciones similares que ayudan a la construcción de los universos ficticios galdosianos: el bloque de clusters que contienen la palabra calle (de la calle de la, por ejemplo) se emplea habitualmente para identificar, además de lugares, a personajes y así caracterizarlos (el carbonero de la calle de la Esperancilla o el prestamista de la calle de San Blas); los que contienen la palabra puerta (a la puerta de la, por ejemplo) suelen funcionar, además de como referencias en la que se sitúan los personajes y en torno a la que se construye el universo ficticio, como 
punto de finalización de una escena, sobre todo cuando los personajes se despiden; finalmente, los que contienen dos referencias espaciales ( $a$ un lado y otro) se asocian frecuentemente con la mirada de los personajes para que presenciemos la escena a través de sus ojos y construyamos la escena en nuestra imaginación. Además, analizados en conjunto, en el uso de estas construcciones también se han detectado funciones relevantes en lo que a la configuración de los universos ficticios se refiere. Como se ha podido comprobar, Galdós se apoya en las referencias espaciales para crear sensación de movimiento y también para dotar de ritmo a la acción mediante la creación de impresión de simultaneidad entre lo que está ocurriendo en el plano discursivo y los aspectos que rodean a las conversaciones.

Todos estos aspectos, en suma, revelan hábitos en el estilo de Galdós que, tal vez por su dispersión en la obra del autor — de más de setenta novelas y casi seis millones y medio de palabras-, no ha sido posible analizar de forma metódica con anterioridad. En este sentido, además de presentar un estudio de la figura de Galdós desde un punto de vista estilístico, este trabajo pretende servir asimismo como muestra de la validez de la estilística de corpus en el análisis de textos literarios en lengua española y abrir así nuevas vías de análisis que hasta ahora no han sido exploradas en la exégesis de autores en este idioma.

\section{REFERENCIAS BIBLIOGRÁFICAS}

ÁLVAREZ MÉNDEZ, N. (2002). Espacios narrativos. León: Universidad de León.

ARROYO DÍEZ, V. (2011). Aspectos espaciales y visuales en las primeras novelas contemporáneas Benito Pérez Galdós y su repercusión en la novela española actual. Tesis doctoral inédita. Valladolid: Universidad de Valladolid. 
CANTOS GÓMEZ, P. Y SÁNCHEZ, A. (2001). "Lexical Constellations: What Collocates Fail to Tell". International Journal of Corpus Linguistics 6.2, 199-228 (también en https://benjamins. com/\#catalog/journals/ijcl.6.2.02can/details [30/08/2018]).

GARRIDO DOMÍNGUEZ, A. (1993). El texto narrativo. Madrid: Síntesis. GONZÁLEZ, J. R. (1998). "Nueva meditación del marco: ciudad y literatura en el fin del siglo". Ínsula 613, 30-33.

GULLÓN, R. (1980). Técnicas de Galdós. Madrid: Taurus.

LAMBERT, M. (1981). Dickens and the Suspended Quotation. New Haven: Yale University Press.

LÓPEZ-LANDY, R. (1979). Espacios narrativos en la novela de Galdós. Madrid: Ediciones Cultura Hispánica del Centro Iberoamericano de Cooperación.

MAHLBERG, M. (2013). Corpus Stylistics and Dickens's Fiction. London: Routledge.

(2016). "Corpus stylistics". En The Bloomsbury Companion to Stylistics, V. Sotirova (ed.), 139-156. Londres y Nueva York: Bloomsbury.

MAHLBERG, M.; SMITH, C. \& PRESTON, S. (2013). "Phrases in literary contexts: Patterns and distributions of suspensions in Dickens's novels". International Journal of Corpus Linguistics 18.1, 35-56 (también en https://benjamins.com/\#catalog/journals/ ijcl.18.1.05mah/details [30/08/2018]).

MARTÍNEZ CARAZO, C. (2006). De la visualidad literaria a la visualidad filmica. La Regenta de Leopoldo Alas Clarín. Gijón: Llibros del Pexe.

MATZAT, W. (2007). Espacios y discursos en la novela española: del realismo a la actualidad. Frankfurt: Iberoamericana Vervuert.

PÉREZ GALDÓS, B. (1874). Tormento. Madrid: Imprenta y Litografía de La Guirnalda. Consultada a partir de la edición digitalizada por la Biblioteca Virtual Miguel de Cervantes en 2000: http://www. cervantesvirtual.com/obra/tormento--0/ [30/08/2018]. 
(1875a). El 19 de Marzo y el 2 de Mayo. Madrid: Imprenta de Noguera a cargo de M. Martínez. Consultada a partir de la edición digitalizada por la Biblioteca Virtual Miguel de Cervantes en 2001: http://www.cervantesvirtual.com/obra/el-19-de-marzo-y-el-2-demayo--0/ [30/08/2018].

(1875b). El equipaje del Rey José. Madrid: Imprenta y Litografía de La Guirnalda. Consultada a partir de la edición digitalizada por la Biblioteca Virtual Miguel de Cervantes en 2001: http:// www.cervantesvirtual.com/obra/el-equipaje-del-rey-jose--0/ [30/08/2018].

(1876a). 7 de Julio. Madrid: Imprenta de José María Pérez. Consultada a partir de la edición digitalizada por la Biblioteca Virtual Miguel de Cervantes en 2001: http://www.cervantesvirtual.com/obra/7-dejulio--0/ [30/08/2018].

(1876b). Napoleón en Chamartín. Madrid: Imprenta de José María Pérez. Consultada a partir de la edición digitalizada por la Biblioteca Virtual Miguel de Cervantes en 2001: http://www.cervantesvirtual. com/obra/napoleon-en-chamartin--0/ [30/08/2018].

(1879). Los apostólicos. Madrid: Imprenta y Litografía de La Guirnalda. Consultada a partir de la edición digitalizada por la Biblioteca Virtual Miguel de Cervantes en 2001: http://www. cervantesvirtual.com/obra/los-apostolicos--0/ [30/08/2018].

(1883). El doctor Centeno. Madrid: Imprenta y Litografía de La Guirnalda. Consultada a partir de la edición digitalizada por la Biblioteca Virtual Miguel de Cervantes en 2001: http://www. cervantesvirtual.com/obra/el-doctor-centeno--0/ [30/08/2018].

(1887). Fortunata y Jacinta. Madrid: Imprenta de La Guirnalda. Consultada a partir de la edición digitalizada por la Biblioteca Virtual Miguel de Cervantes en 2001: http://www.cervantesvirtual. com/obra/fortunata-y-jacinta-dos-historias-de-casadas--0/ [30/08/2018].

(1888). Miau. Madrid: Imprenta de La Guirnalda. Consultada a 
partir de la edición digitalizada por la Biblioteca Virtual Miguel de Cervantes en 2001: http://www.cervantesvirtual.com/obra/miau-$0 /[30 / 08 / 2018]$.

(1891). Ángel Guerra. Madrid: Administración de La Guirnalda y Episodios Nacionales. Consultada a partir de la edición digitalizada por la Biblioteca Virtual Miguel de Cervantes en 2003: http://www. cervantesvirtual.com/obra/angel-guerra--0/ [30/08/2018].

(1897). Misericordia. Madrid: Viuda e Hijos de Tello. Consultada a partir de la edición digitalizada por la Biblioteca Virtual Miguel de Cervantes en 2001: http://www.cervantesvirtual.com/obra/ misericordia--0/ [30/08/2018].

(1899). Vergara. Madrid: Viuda e Hijos de Tello. Consultada a partir de la edición digitalizada por la Biblioteca Virtual Miguel de Cervantes en 2001: http://www.cervantesvirtual.com/obral vergara--0/ [30/08/2018].

(1900). Montes de Oca. Madrid: Viuda e Hijos de Tello. Consultada a partir de la edición digitalizada por la Biblioteca Virtual Miguel de Cervantes en 2001: http://www.cervantesvirtual.com/obra/montesde-oca--0/ [30/08/2018].

(1904). O’Donnell. Madrid: Viuda e Hijos de Tello. Consultada a partir de la edición digitalizada por la Biblioteca Virtual Miguel de Cervantes en 2001: http://www.cervantesvirtual.com/obra/ odonnell--0/ [30/08/2018].

(1909). La desheredada. Madrid: Librería de Perlado, Páez y Compañía. Consultada a partir de la edición digitalizada por la Biblioteca Virtual Miguel de Cervantes en 2001: http://www. cervantesvirtual.com/obra/la-desheredada--0/ [30/08/2018].

(1910). Amadeo I. Madrid: Perlado, Páez y Compañía. Consultada a partir de la edición digitalizada por la Biblioteca Virtual Miguel de Cervantes en 2001: http://www.cervantesvirtual.com/obra/ amadeo-i--0/ [30/08/2018].

(1911). La Primera República. Madrid: Perlado, Páez y Compañía. 
Consultada a partir de la edición digitalizada por la Biblioteca Virtual Miguel de Cervantes en 2001: http://www.cervantesvirtual. com/obra/la-primera-republica--0/ [30/08/2018].

SCOTT, M. (2013). WordSmith Tools Manual. Version 6.0. Liverpool: Lexical Analysis Software.

(2016). WordSmith Tools version 7. Stroud: Lexical Analysis Software.

STUBBS, M. (2005). "Conrad in the computer: Examples of quantitative stylistics methods". Language and Literature 14.1, 5-24 (también en http://journals.sagepub.com/doi/abs/10.1177/0963947005048873 [30/08/2018]).

TAMBLING, E. (2013). "Dickens and Galdós". En The reception of Charles Dickens in Europe, M. Hollington (ed.), 191-196. Londres: Bloomsbury.

TOGNINI-BONELLI, E. (2001). Corpus Linguistics at Work. Amsterdam: John Benjamins.

VALLES CALATRAVA, J. R. (2008). Teoría de la narrativa. Una perspectiva sistemática. Madrid: Iberoamericana Vervuert.

ZORAN, G. (1984). "Towards a Theory of Space in Narrative". Poetics Today 5.2, 309-335.

ZUBIAURRE, M. T. (2000). El espacio en la novela realista. México: Fondo de Cultura Económica.

Recibido el 14 de marzo de 2018.

Aceptado el 4 de septiembre de 2018. 